\title{
Proteomic analysis of protein interactions between Eimeria maxima sporozoites and chicken jejunal epithelial cells by shotgun LC-MS/MS
}

\author{
Jingwei Huang, Tingqi Liu, Ke Li, Xiaokai Song, Ruofeng Yan, Lixin Xu and Xiangrui Li*
}

\begin{abstract}
Background: Eimeria maxima initiates infection by invading the jejunal epithelial cells of chicken. However, the proteins involved in invasion remain unknown. The research of the molecules that participate in the interactions between E. maxima sporozoites and host target cells will fill a gap in our understanding of the invasion system of this parasitic pathogen.

Methods: In the present study, chicken jejunal epithelial cells were isolated and cultured in vitro. Western blot was employed to analyze the soluble proteins of E. maxima sporozoites that bound to chicken jejunal epithelial cells. Co-immunoprecipitation (co-IP) assay was used to separate the E. maxima proteins that bound to chicken jejunal epithelial cells. Shotgun LC-MS/MS technique was used for proteomics identification and Gene Ontology was employed for the bioinformatics analysis.

Results: The results of Western blot analysis showed that four proteins bands from jejunal epithelial cells co-cultured with soluble proteins of E. maxima sporozoites were recognized by the positive sera, with molecular weights of 70, 90, 95 and $130 \mathrm{kDa}$. The co-IP dilutions were analyzed by shotgun LC-MS/MS. A total of 204 proteins were identified in the E. maxima protein database using the MASCOT search engine. Thirty-five proteins including microneme protein 3 and 7 had more than two unique peptide counts and were annotated using Gene Ontology for molecular function, biological process and cellular localization. The results revealed that of the 35 annotated peptides, 22 (62.86\%) were associated with binding activity and 15 (42.86\%) were involved in catalytic activity.
\end{abstract}

Conclusions: Our findings provide an insight into the interaction between E. maxima and the corresponding host cells and it is important for the understanding of molecular mechanisms underlying E. maxima invasion.

Keywords: Eimeria maxima, Invasion, Chicken, Jejunal epithelial cells, Parasite-host interaction

\section{Background}

Coccidiosis is an enteric disease, caused by the unicellular parasite Eimeria, and a major problem in the poultry industry [1]. Eimeria maxima is one of the most prevalent Eimeria species that causes substantial economic losses worldwide [2]. Eimeria maxima undergoes schizogony and gametogony after the invasion of jejunal epithelial cells and subsequently occupies the cell cytoplasm [3]. Although an in vitro model has been developed to study

\footnotetext{
* Correspondence: lixiangrui@njau.edu.cn

College of Veterinary Medicine, Nanjing Agricultural University, 1 Weigang, Nanjing, Jiangsu 210095, People's Republic of China
}

the invasion of Madin-Darby bovine kidney (MDBK) cells by sporozoites [4], the mechanisms by which infective sporozoites and merozoites recognize, invade and migrate within the intestinal epithelium remain unknown. However, the establishment of E. maxima in the appropriate habitat is important in host infection.

Host-cell invasion by apicomplexan parasites is a unique process dependent on gliding motility and requires a transmembrane link between the parasite cytoskeleton and the host cell [5]. Many proteins are secreted by the parasite to help it penetrate the defense barriers, invade the host cells and avoid the immune attack of the host [6]. 
An event shared by these processes is the appropriate engagement and disengagement of specific receptors upon the regulated apical release of invasion proteins from parasite secretory organelles (micronemes, rhoptries) [7]. Therefore, the identification of soluble protein components of the invasive sporozoite stage functioning at the interface between E. maxima and host intestinal epithelial cells is crucial to understand the mechanism of parasite invasion and identify possible vaccine target.

In this study, Western blot, co-IP, and shotgun LC-MS/ MS were combined to characterize E. maxima sporozoite proteins binding to the chicken jejunal epithelial cells and to screen the proteins related to invasion.

\section{Methods}

\section{Parasites and animals}

The parasites E. maxima and Chinese yellow chickens used in this study were obtained and kept in conditions as previously described [8].

\section{Collection and purification of sporozoites}

Sporozoites from E. maxima sporulated oocysts were purified using DE-52 anion exchange columns (GE Whatman, Pleasanton, USA) according to a protocol described previously [9]. Finally, the collected sporozoites were stored in liquid nitrogen before use.

\section{Soluble antigens of $E$. maxima}

The soluble proteins of E. maxima were prepared following a protocol described previously with slight modifications [2]. Briefly, the pellet of $5 \times 10^{8}$ sporozoites was dissolved in $2 \mathrm{ml}$ of PBS containing $0.5 \%$ Triton X-100 and $1 \%$ protease inhibitor cocktail set III (Millipore, Burlington, USA) and was disrupted by ultrasound in an ice bath. Then, the supernatant proteins were separated by high-speed centrifugation $\left(14,000 \times \mathrm{g}, 30 \mathrm{~min}, 4{ }^{\circ} \mathrm{C}\right)$, aliquoted and stored at $-70{ }^{\circ} \mathrm{C}$ until used in co-culture with chicken jejunal epithelial cells. Twenty micrograms of E. maxima soluble proteins were subjected to SDS-PAGE analysis using a $12 \%$ gel.

\section{Antisera against soluble proteins of $E$. maxima}

The polyclonal sera against E. maxima soluble proteins were prepared according to a method described previously [10]. Briefly, approximately $100 \mu \mathrm{g}$ of soluble proteins of E. maxima sporozoites were mixed with Freund's complete adjuvant (Invitrogen, Carlsbad, USA) and injected intraperitoneally into naïve SD rats (twoweek-old, female). After a two-week interval, $100 \mu \mathrm{g}$ of soluble proteins of E. maxima sporozoites in Freund's incomplete adjuvant (Invitrogen) were intraperitoneally injected. In total three boosters were conducted. The serum was collected 14 days after the last injection and stored at $-70{ }^{\circ} \mathrm{C}$ until use. In addition, sera collected before proteins injection was used as a negative control.

\section{Isolation of chicken jejunal epithelial cells}

Chicken jejunal epithelial cells were isolated using a method described previously $[11,12]$. Coccidian-free chickens were immerged for $5 \mathrm{~min}$ in $70 \%$ ethanol after they were slaughtered and the blood was drained. After that, the jejunums were dislodged and put into Hanks' balanced salt solution (HBSS, PAA, Linz, Austria). Then the jejunums were washed by HBSS until the mucus was completely removed. Following dissection of the mucosa into small strips, the strips were put into $1 \mathrm{mM}$ DTT (Sigma-Aldrich, St. Louis, USA) in $50 \mathrm{HBSS}(30 \mathrm{~min}$ at ambient temperature). Sequentially, the mucosal strips were incubated in $1 \mathrm{mM}$ EDTA (Sigma) for $10 \mathrm{~min}$ at $37^{\circ} \mathrm{C}$. Mucosal strips were briefly rinsed in HBSS (to eliminate already detached jejunal epithelial cells), transferred to fresh HBSS at ambient temperature and followed by $5-10$ vigorous shakes of the container. This procedure leads to instant detachment of jejunal epithelial cells in a full-length crypt formation. After rapid removal of the mucosal strips by passing the solution over a coarse mesh $(80 \mu \mathrm{m}$, Rotilabo sieve, Carl Roth GmbH, Karlsruhe, Germany), rapid purification of detached jejuna epithelial cells was achieved by usage of a mesh filter ( $400 \mu \mathrm{m}$ pore size, Sefer, Kansas City, KS, USA) fixed with tape to a plastic ring ( $5 \mathrm{~cm}$ diameter, 2 $\mathrm{cm}$ height and $3 \mathrm{~mm}$ thickness). The suspension containing jejunal epithelial cells crypts was gently but rapidly passed over the mesh to separate the jejunal epithelial cells crypts from single cells (erythrocytes, leukocytes, fibroblast, etc.) which easily passed through the filter. The filter was then rapidly inverted and purified intact jejuna epithelial cells crypts were immediately washed out with culture medium (see below) at ambient temperature. The jejunal epithelial cells crypt solution was then rapidly transferred to the ECM-coated culture dish and cultured at $41{ }^{\circ} \mathrm{C}$ and $\mathrm{CO}_{2}$ for $1.5 \mathrm{~h}$. The non-adherent cells were collected for identification of the jejunal epithelial cells and co-culture with the soluble proteins of $\mathrm{E}$. maxima sporozoite.

Co-culture of jejunal epithelial cells and soluble proteins of E. maxima sporozoites

The collected jejunal epithelial cells were suspended in an advanced culture medium [DMEM containing 5\% fetal bovine serum (FBS), $100 \mathrm{U} / \mathrm{ml}$ penicillin and 100 $\mathrm{mg} / \mathrm{ml}$ streptomycin, $20 \mathrm{mmol} / \mathrm{l}$ HEPES, $1 \mathrm{mmol} / \mathrm{l}$ Supplement, $5 \mathrm{mg} / \mathrm{l}$ bovine pituitary extract, and 0.01 $\mathrm{mg} / \mathrm{l}$ epidermal growth factor (EGF) (all products were from Gibco Life technologies, New York, USA)]. Subsequently, the cells were seeded in a 6-well plate (Corning, Corning, NY, USA; $1 \times 10^{6}$ cells per well) and cultured at $41{ }^{\circ} \mathrm{C}$ in an atmosphere of $95 \%$ air and $5 \% \mathrm{CO}_{2}$ for $2 \mathrm{~h}$. Following sterilization using a $0.22 \mu \mathrm{m}$ filter (Millipore), the soluble proteins (final concentration of $100 \mu \mathrm{g} / \mathrm{ml}$ ) of E. maxima sporozoites were added to the culture medium and incubated for $1 \mathrm{~h}$. The jejunal epithelial cells were washed four 
times with $0.1 \mathrm{M}$ PBS buffer (pH 7.8) after removal of the culture medium.

\section{Extraction of proteins from co-cultured jejunal epithelial cells}

The washed cells were mixed with $0.2 \mathrm{ml}$ cell lysis $(\mathrm{pH} 7$. 2, $60 \mathrm{mM}$ Tris- $\mathrm{HCl} ; 2 \%$ sodium dodecyl sulfate; SDS) containing $1 \%$ protease inhibitor cocktail set III (Millipore), subjected to three freeze-thaw cycles at $-80{ }^{\circ} \mathrm{C}$ and $37{ }^{\circ} \mathrm{C}$, and sonicated in an ice bath as described above. The soluble supernatant was filtered through a $0.22 \mu \mathrm{m}$ mesh filter, aliquoted and stored at $-70^{\circ} \mathrm{C}$ before use.

\section{SDS-PAGE and Western blot analysis}

Twenty micrograms of protein samples from co-cultured jejunal epithelial cells were subjected to SDS-PAGE using a routine method described elsewhere [13].

Western blot was employed to analyze the soluble proteins of E. maxima sporozoite that bound to chicken jejunal epithelial cells. Briefly, the protein samples were separated using a 12\% SDS-PAGE gel as described above and transferred to a nitrocellulose membrane (Millipore) using Semi-Dry Transfer Cell (Bio-Rad, Hercules, USA) according to the manufacturer's instructions. Rat antiserum against the soluble proteins of E. maxima sporozoite (dilution 1:200) was used as the primary antibody and horseradish peroxidase (HRP)-conjugated goat anti-rat IgG (dilution 1:2000, Sigma) was used as the secondary antibody. The bound antibodies were detected using Pierce DAB Substrate Kit (Thermo Fisher Scientific, Waltham, USA) according to the manufacturer's instructions.

\section{Co-immunoprecipitation (co-IP) assay}

The co-IP assay was conducted according to the manufacturer's instructions (Protein A/G PLUS-Agarose: sc-2003, Santa Cruz Biotechnology Inc., Delaware Ave Santa Cruz, USA). Briefly, $5 \times 10^{7}$ washed jejunal epithelial cells were incubated with $3 \mathrm{ml}$ of ice-cold RIPA buffer (Vazyme Biotech Co. Ltd., Nanjing, China) containing 1\% protease inhibitor cocktail set III (Millipore) for $15 \mathrm{~min}$ on ice and were disrupted by repeated passage through a 21-gauge needle. Cellular debris was removed by centrifugation at $13,000 \times g$ for $10 \mathrm{~min}$ at $4{ }^{\circ} \mathrm{C}$, and the supernatant was transferred to a microcentrifuge tube on ice. One microgram of normal rat polyclonal IgG and $20 \mu \mathrm{l}$ of suspended Protein A/G-Agarose (25\%, V/V) was added to the supernatant and incubated at $4{ }^{\circ} \mathrm{C}$ for $1 \mathrm{~h}$ on a rotator. The supernatant was separated by centrifugation at $1,000 \times g$ for $5 \mathrm{~min}$ at $4{ }^{\circ} \mathrm{C}$ and transferred to a microcentrifuge tube on ice. Two micrograms of the primary antibody was added to the supernatant and incubated at $4{ }^{\circ} \mathrm{C}$ for $2 \mathrm{~h}$. Twenty microliters of the suspended Protein A/G-Agarose $(25 \%, V / V)$ was added to the supernatant and incubated at $4{ }^{\circ} \mathrm{C}$ overnight. The immunoprecipitates were collected by centrifugation at $1,000 \times g$ for $5 \mathrm{~min}$ at $4{ }^{\circ} \mathrm{C}$ and the supernatant was carefully aspirated and discarded. The immunoprecipitates were gently washed four times with $1 \mathrm{ml}$ of ice-cold RIPA buffer (Vazyme biotech Co. Ltd.) containing $1 \%$ protease inhibitor cocktail set III (Millipore). After the final wash, $40 \mu \mathrm{l}$ of $2 \times$ electrophoresis loading buffer (Vazyme biotech Co. Ltd.) was used to resuspend the pellet. The immunoprecipitated samples were boiled for 2-3 min and stored at $-20^{\circ} \mathrm{C}$ for protein identification.

\section{Liquid chromatography and tandem mass spectrometry}

The immunoprecipitates were sent to Applied Protein Technology Co. Ltd. (Shanghai, China) and the assays were conducted on a $\mathrm{Q}$ Exactive mass spectrometer coupled to Easy nLC (Thermo Fisher Scientific) using a routine method [14]. Briefly, trypsin-digested peptides (approximately $30 \mu \mathrm{g}$ ) were trapped and desalted on Zorbax 300SB-C18 peptide traps (Agilent Technologies, Wilmington, DE, USA) and separated on a C18-reversed phase column $(0.15 \times 150 \mathrm{~mm}$, Column Technology Inc., Fremont, CA, USA). The Easy nLC system (Thermo Fisher Scientific) was used to deliver mobile phases A ( $0.1 \%$ formic acid in HPLC-grade water) and B (0.1\% formic acid in $84 \%$ acetonitrile) with a linear gradient of $4-50 \%$ B (50 min), $50-100 \%$ B (4 min), and then $100 \%$ B (6 min) at a flow rate of $250 \mathrm{nl} / \mathrm{min}$. To acquire the MS data, a datadependent top ten method was used, in which the ten most abundant precursor ions were selected for HCD fragmentation.

\section{Protein identification and annotation}

MS/MS spectra were searched using MASCOT 2.2 (Matrix Science, London, UK) against the non-redundant International Protein Index Eimeria maxima sequence database version 3.85 (released at May 2014; 6127 sequences) from the European Bioinformatics Institute (http://www.ebi.ac.uk/). The following parameters were used for protein identification: peptide mass tolerance, $20 \mathrm{ppm} ; \mathrm{MS} / \mathrm{MS}$ tolerance, $0.1 \mathrm{Da}$; enzyme, trypsin; number of missed cleavages, 2; fixed modification, carbamidomethyl (C); variable modification, oxidation $(\mathrm{M})$; score $>20$.

\section{InterProScan annotation and Gene Ontology (GO) categories}

InterProScan software (http://www.ebi.ac.uk/interpro/ search/sequence-search) was used to perform protein sequences searches against InterPro databases and identify protein signatures [15]. The matched terms were output in RAW format. The RAW data were subjected to GO categories using the Web Gene Ontology Annotation Plot (WEGO) [16]. The three groups of datasets were simultaneously subjected to online analysis (http://wego. genomics.org.cn/) and were compared in one graph. The 
$P$-values were calculated using the Pearson's Chi-square test.

\section{Results}

SDS-PAGE and Western blot analysis of soluble proteins of E. maxima sporozoites

The soluble proteins of $E$. maxima sporozoites were analyzed by SDS-PAGE (Fig. 1a). The molecular weight of the protein bands ranged from 10 to $170 \mathrm{kDa}$.

Western blot analysis indicated that the soluble proteins were identified by the antisera of rats experimentally immunized with the parasite proteins (Fig. 1b). However, these soluble proteins could not be identified by the pre-immune rat sera.

\section{SDS-PAGE and Western blot analysis of proteins of co- cultured cells}

The fractionated proteins of jejunal epithelial cells cocultured with soluble proteins of E. maxima sporozoites were analyzed by SDS-PAGE and stained with Coomassie brilliant blue. The molecular weight of the protein bands ranged from 17 to $170 \mathrm{kDa}$ (Fig. 2).

Western blot analysis indicated that rats were successfully seroconverted after experimentally immunized with soluble proteins of E. maxima sporozoites. However, no protein bands were detected using pre-immune rat sera. On the other hand, proteins of jejunal epithelial cells co-cultured

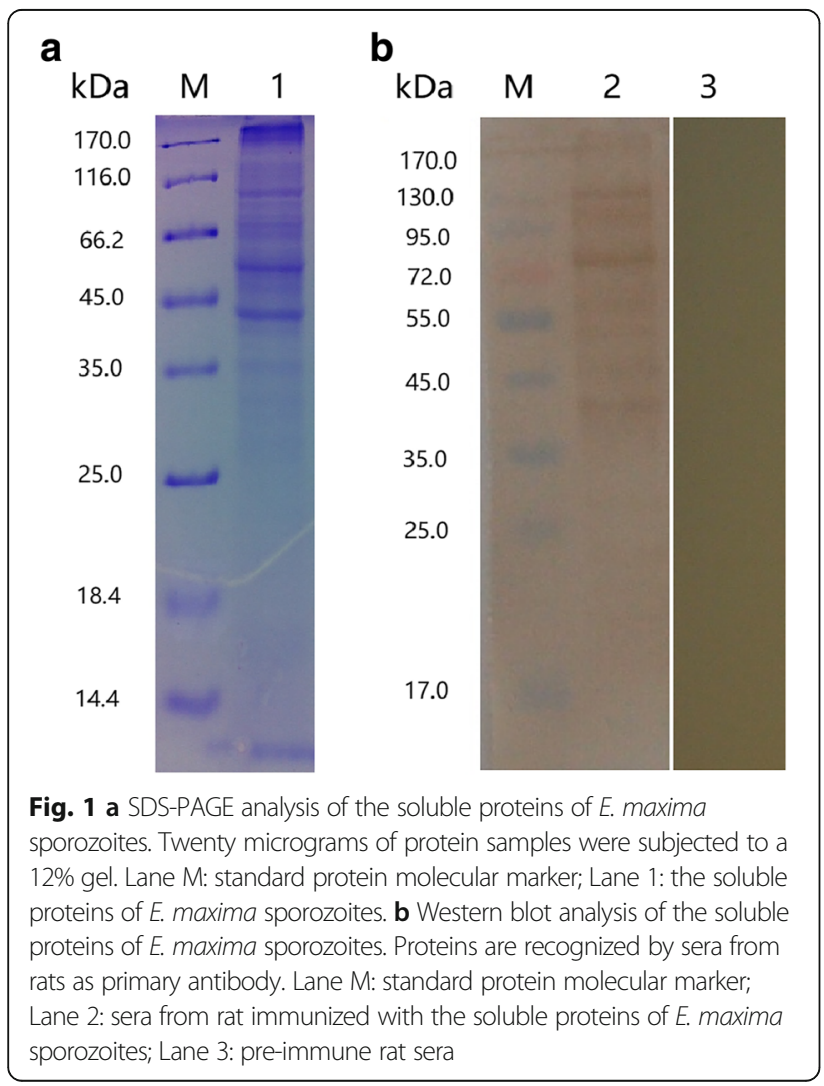

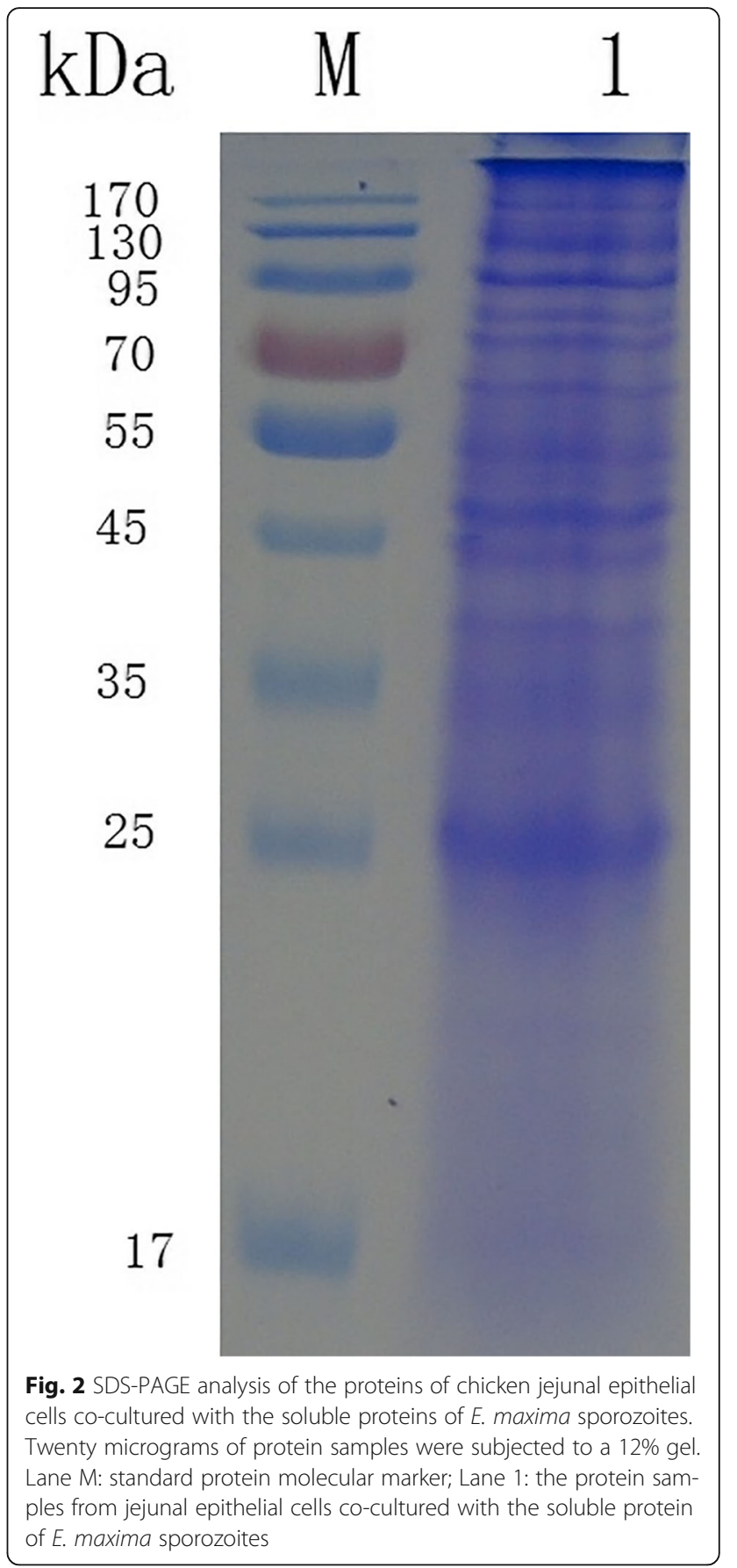

with PBS buffer as negative control were not able to be detected by antisera of rats immunized with the target proteins. Four protein bands were recognized by the antisera, with molecular weights of 70, 90, 95 and $130 \mathrm{kDa}$ (Fig. 3).

\section{LC-MS/MS analysis of proteins of E. maxima sporozoites binding to jejunal epithelial cells}

After analysis by QE shotgun LC-MS/MS [17], 204 nonredundant proteins were identified by searching the $E$. maxima database in Uniprot and NCBI (Additional file 1: 


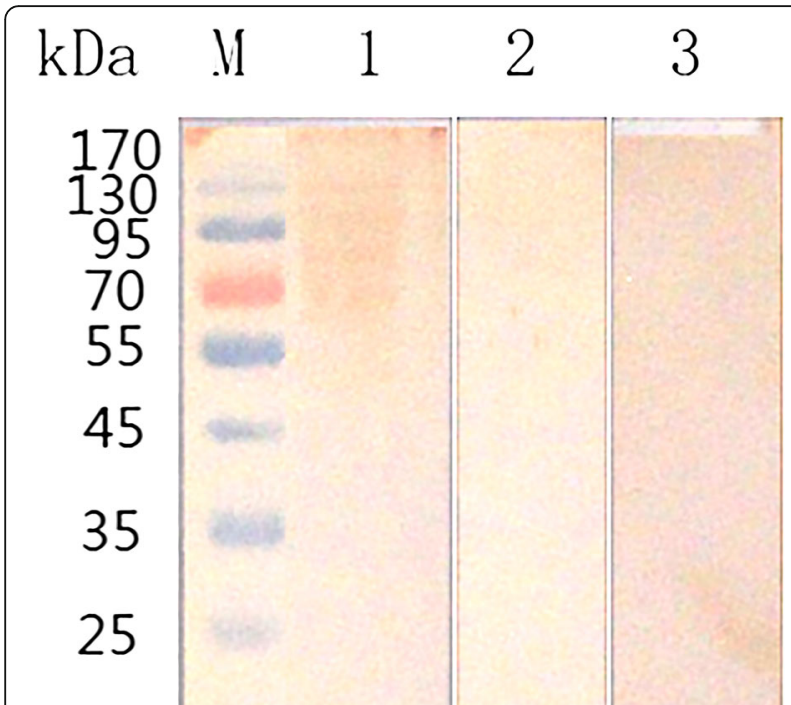

Fig. 3 Western blot analysis of proteins of chicken jejunal epithelial cells co-cultured with the soluble proteins of E. maxima sporozoites. Proteins are recognized by sera from rats as primary antibody. Lane M: standard protein marker; Lane 1: Iysis of chicken jejunal epithelial cells cultured with E. maxima sporozoites soluble proteins detected by sera from rats immunized with soluble proteins of E. maxima sporozoites; Lane 2: Iysis of chicken jejunal epithelial cells cultured with PBS buffer detected by sera from rats immunized with soluble proteins of E. maxima sporozoites; Lane 3: lysis of chicken jejunal epithelial cells cultured with E. maxima sporozoites soluble proteins detected by pre-immune rat sera

Table S1). Only 35 proteins contained more than two unique peptide counts (Additional file 2: Table S2). Among these, microneme proteins (MIC2, MIC3 and MIC7), aldolase, and elongation factors were associated with invasion; 56 and $82 \mathrm{kDa}$ gametocyte antigens were involved in the formation of the oocyst wall and development of the parasite life-cycle, and actin and tubulin were related to parasite movement. Other proteins were involved in cell signaling, including 14-3-3 superfamily proteins. Some of the identified proteins were related to the metabolism of phosphate, lipids, and nucleic acids, including aldolase, enolase 2, RAS small GTPase, and the alpha and beta chains of ATP synthase. Moreover, potential housekeeping genes were significantly enriched, including actin, tubulin, histone, and heat shock proteins. Three hypothetical proteins that had not been characterized were also present in this analysis (Table 1).

\section{Functional categories of proteins of E. maxima sporozoites binding to jejunal epithelial cells}

The 35 proteins with more than two unique peptide counts were analyzed using Gene Ontology (GO) and categorized into biological process, molecular function, and cellular component. The annotation by biological process (Fig. 4a) indicated that 22 proteins were related to cellular processes (GO 0009987), metabolic processes (GO 0008152), single- organism processes (GO 0044699), response to stimulus (GO 0050896), cellular component organization or biogenesis (GO 0071840), localization (GO 0051179) and biological regulation (GO 0065007). Other proteins were involved in multicellular organismal processes (GO 0032501), signaling (GO 0023052), multi-organism processes (GO 0051704) and locomotion (GO 0040011).

Five subcategories were assigned to molecular function (Fig. 4b). Binding activity (GO 0005488, 22 proteins, 62. $86 \%$ of the 35 annotated peptides) and catalytic activity (GO 0003824, 15 proteins, 42.86\% of the 35 annotated peptides) were the most representative molecular function categories. The remaining proteins were related to enzyme regulatory activity (GO 0030234), structural molecule activity (GO 0005198) and transport activity (GO 0005215).

The cellular components ontology (Fig. 4c) refers to the cellular regions where a gene product is active [18]. Following this category, of the identified proteins, 16 were present in the cell (GO 0005623), 11 in the macromolecular complex (GO 0032991), 10 in the organelle (GO 0043226), five in the membrane (GO 0016020), one in the extracellular region (GO 0005576), one in the symplast (GO 0055044) and one in cell junctions (GO 0030054).

Most cellular and metabolic processes were related to synthesis and degradation of macromolecules, particularly nucleotides, carbohydrates and proteins, and these processes might be associated with the invasion and development of E. maxima.

\section{Discussion}

The coccidian parasite E. maxima is one of the seven Eimeria species which infect intestinal epithelial cells of chicken [19]. However, to date it is unclear why E. maxima preferentially invades and survives in jejunal epithelial cells of chicken small intestines. In the present study, the proteins of E. maxima sporozoites that bound to these epithelial cells were analyzed using the co-IP assay and QE shotgun LC-MS/MS analysis. Our results highlighted the importance of exploring the location specificity of E. maxima.

All invasive forms of apicomplexans, including Eimeria spp., possess a unique complex of organelles, the apical complex, located at the anterior end of the organism. The apical complex comprises rhoptries, micronemes, dense granules, as well as an apical assembly of cytoskeleton-associated structures including the conoid, polar/apical rings and micro tubular protrusions [20]. Most of these organelles secret proteins involved in parasite attachment, invasion and intercellular development $[21,22]$. Microneme organelle proteins (MICs) are critical for parasite motility and host cell invasion [23]. MICs secreted in the early stages of invasion, participate in parasite attachment to the host cells and subsequent 
Table 1 Proteins of E. maxima that bound to chicken jejunal epithelial cells identified by shotgun LC-MS/MS with more than two unique peptide counts

\begin{tabular}{|c|c|c|c|c|c|c|c|}
\hline Protein description & $\begin{array}{l}\text { Accession } \\
\text { number }\end{array}$ & $\begin{array}{l}\text { Unique pep } \\
\text { count } \mathrm{MH}+\end{array}$ & $\begin{array}{l}\text { Pep count } \\
\text { (sequence) }\end{array}$ & $\begin{array}{l}\text { Cover } \\
\text { percent (\%) }\end{array}$ & $\begin{array}{l}\text { Theor MW } \\
\text { (kDa) }\end{array}$ & Theor pl & $\begin{array}{l}\text { MASCOT } \\
\text { score }\end{array}$ \\
\hline Lactate dehydrogenase & gi|557194155 & 4 & 4 & 16.97 & 35.94 & 6.60 & 60.84 \\
\hline 14-3-3 protein & gi|557193108 & 6 & 6 & 23.43 & 32.55 & 4.80 & 63.59 \\
\hline Elongation factor 1-alpha & gi|557198794 & 3 & 4 & 6.44 & 49.36 & 9.16 & 63.00 \\
\hline Elongation factor 2 & gi|557157066 & 3 & 3 & 3.37 & 93.28 & 5.99 & 49.12 \\
\hline Fructose-bisphosphate aldolase & gi|557193254 & 3 & 3 & 13.13 & 38.77 & 8.33 & 62.10 \\
\hline ATP synthase beta chain & gi|557185041 & 3 & 3 & 4.80 & 72.32 & 6.22 & 47.06 \\
\hline 18 kda cyclophilin & gi|557198725 & 3 & 3 & 23.28 & 20.35 & 6.97 & 54.77 \\
\hline Hypothetical protein, conserved & gi|557206977 & 3 & 3 & 11.67 & 32.25 & 4.58 & 53.47 \\
\hline Heat-shock protein 60 & gi|557243650 & 3 & 3 & 8.73 & 59.93 & 5.86 & 53.46 \\
\hline B-block-binding subunit of tfiiic protein & gi|557157037 & 2 & 9 & 0.46 & 34.63 & 6.76 & 44.91 \\
\hline Polyubiquitin & gi|557209304 & 2 & 5 & 11.54 & 17.46 & 8.01 & 39.48 \\
\hline RAS small GTpase & gi|557167708 & 2 & 3 & 8.33 & 22.94 & 7.64 & 71.94 \\
\hline Heat-shock protein, related & gi|557184708 & 5 & 5 & 7.65 & 71.41 & 5.16 & 80.62 \\
\hline Microneme protein 7 & gi|343094702 & 2 & 2 & 15.12 & 18.24 & 4.62 & 57.25 \\
\hline Tubulin beta chain & gi|343480759 & 2 & 2 & 6.01 & 49.92 & 4.78 & 76.11 \\
\hline ATP synthase alpha & gi|557156948 & 2 & 2 & 6.90 & 21.68 & 7.63 & 43.05 \\
\hline $\begin{array}{l}\text { SWI/SNF-related matrix-associated } \\
\text { actin-dependent regulator of chromatin }\end{array}$ & gi|557158395 & 2 & 2 & 1.51 & 11.91 & 5.64 & 41.06 \\
\hline ADP ribosylation factor 1 & gi|557167614 & 2 & 2 & 11.40 & 20.80 & 6.10 & 56.12 \\
\hline Protein emb isoform, related & gi|557184804 & 2 & 2 & 0.61 & 23.58 & 5.80 & 35.63 \\
\hline Hypothetical protein, conserved & gi|557168315 & 2 & 2 & 0.38 & 38.08 & 6.14 & 29.12 \\
\hline Hypothetical protein, conserved & gi|557210462 & 2 & 2 & 0.26 & 63.97 & 6.65 & 34.58 \\
\hline 60s ribosomal protein L3 & gi|557210678 & 2 & 2 & 4.90 & 44.11 & 10.41 & 60.01 \\
\hline Heat-shock protein 90 & gi|557188011 & 2 & 2 & 2.94 & 82.69 & 5.08 & 76.54 \\
\hline Microneme protein 2 & gi|334851460 & 5 & 5 & 14.64 & 30.60 & 4.90 & 51.92 \\
\hline Heat-shock protein 70 & gi|557232143 & 2 & 2 & 3.25 & 56.17 & 8.69 & 46.71 \\
\hline Triosephosphate isomerase & gi|557232104 & 2 & 2 & 10.76 & 27.36 & 6.00 & 38.99 \\
\hline Tubulin alpha chain & gi|557210668 & 2 & 2 & 4.98 & 46.69 & 4.90 & 55.07 \\
\hline Microneme protein 3, partial & gi|557237363 & 2 & 2 & 4.58 & 44.33 & 4.65 & 51.70 \\
\hline Coiled-coil domain-containing protein, related & gi|557238523 & 2 & 2 & 0.45 & 26.90 & 4.52 & 26.72 \\
\hline Actin & gi|557207008 & 4 & 15 & 12.77 & 42.01 & 5.00 & 49.73 \\
\hline Histone & gi|19880141 & 4 & 9 & 20.59 & 15.39 & 11.29 & 35.08 \\
\hline 82 kda gametocyte, related & gi|38565038 & 4 & 5 & 9.73 & 66.09 & 5.16 & 101.05 \\
\hline 56 kda gametocyte, related & gi|557167757 & 4 & 5 & 9.03 & 53.30 & 5.00 & 78.91 \\
\hline Enolase 2 & gi|557157196 & 4 & 4 & 9.37 & 49.88 & 5.92 & 42.82 \\
\hline Glyceraldehyde-3-phosphate dehydrogenase & gi|557168185 & 4 & 4 & 11.21 & 36.26 & 7.58 & 74.56 \\
\hline
\end{tabular}

formation of the connection with the parasite actinomyosin system, thereby providing the platform from which an invasion will be initiated [24]. Previous studies proved that microneme secretion was rapidly increased when parasites contacted host cells and parasite invasion was blocked when microneme secretion was chemically inhibited [25, 26]. To date, nine microneme proteins have been reported in Eimeria: microneme proteins 1-7 (MIC 1-7) and apical membrane antigens 1 and 2 (AMA1 and 2) [27]. In recent studies, dual immunofluorescence staining of $E$. tenella microneme 3 (EtMIC3) and 5 (EtMIC5) on fixed and permeabilized sporozoites of E. tenella showed that the parasites were attached to host cells by the apical region, that EtMIC3 


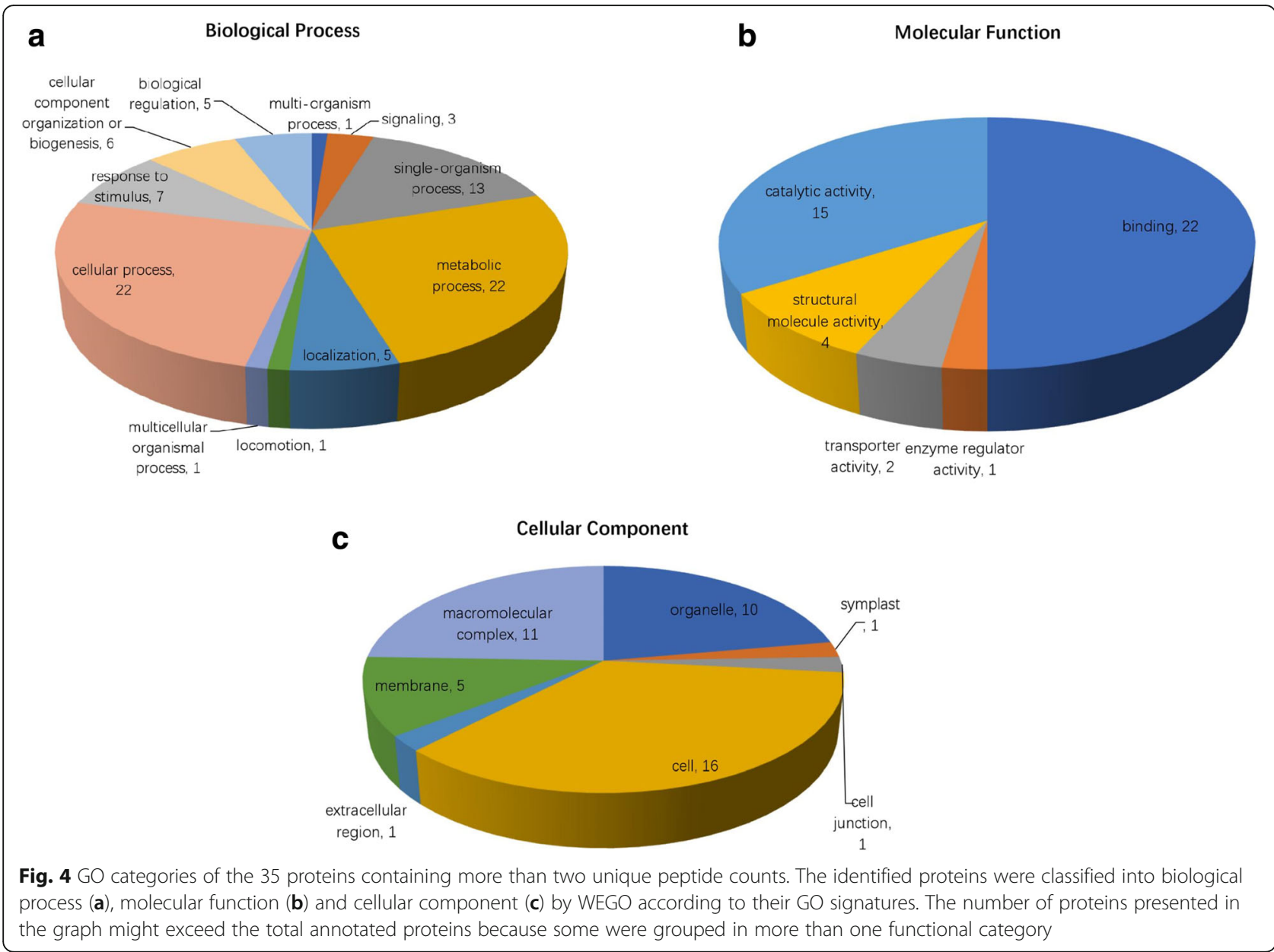

was located primarily at the apical tip of sporozoites [7], it could also bind to sialic acid-containing molecules on the epithelial cell surface of the hosts, and played a key role in cell invasion by sporozoites [7, 28]. Comparably, MIC1 and 13 of T. gondii (TgMIC1 and TgMIC13), and MIC1 of Neospora caninum (NcMIC1) showed similar binding capacity to sialic acid-containing molecules an indication of their roles in parasite-cell attachment regulation [28]. It has also been reported that 11 cysteinerich receptor-like regions similar to the Apple domains of EtMIC5 were associated with parasite membrane and established an important correlation between cytoskeletal elements of parasites and receptors on the surface of host cells [29]. In the present study, three MICs (MIC2, MIC3 and MIC7) of E. maxima were identified. However, their roles during host cell attachment and invasion need to be deeply elucidated.

Several proteins associated with the sexual stage of $E$. maxima, including 56-, 82- and 230-kDa antigens, have been identified as potential vaccine targets for inducing transmission-blocking immunity, and these antigens were later proven to be 56-, $82-$ and $230-\mathrm{kDa}$ gametocyte proteins [30]. Immunization with the 56- and 82-
kDa gametocyte antigens promoted both cellular- and humoral-mediated immunity against experimental coccidiosis and reduced fecal oocyst shedding, which is an important component of the Eimeria life-cycle [19, 30]. In particular, Gam56 and Gam 82 have been implicated in oocyst wall formation [31]. In this respect, the neutralization of Gam56 and Gam82 during the sexual stage could block the development of E. maxima. Previous studies reported that immunization with affinitypurified gametocyte antigens (APGA) from E. maxima, 56-, 82- and 230-kDa gametocyte proteins as subunit vaccines could induce partial protective immunity against E. maxima, E. tenella and E. acervulina infection [32]. In the present study, Gam56 and Gam82 were found among the proteins capable of binding to chicken jejunal epithelial cells. However, the role of gametocyte proteins in invasion needs to be better understood.

Protein 14-3-3 plays important roles in many regulatory processes including mitogenic signal transduction apoptotic cell death, cell cycle control and protein localization $[33,34]$. Furthermore, T. gondii 14-3-3 protein has shown potentiality as a vaccine candidate against toxoplasmosis [35]. Moreover, other studies reported that 14-3-3 protein 
of E. tenella could interact with RNA-binding domain of telomerase reverse transcriptase [36]. In the present study, 14-3-3 protein of E. maxima bound to chicken jejunal epithelial cells, indicating the possible role that might be played by this protein during host cell invasion.

It is well known that all apicomplexan parasites share a unique host cell invasion mechanism that is dependent on actin [37, 38]. Actin filaments are composed of actin and other actin-binding proteins. These structures are highly dynamic in non-muscle cells and play critical roles in many cellular processes, including cell migration [39], cytokines and polarized growth [40, 41]. The actin cytoskeleton is involved in protein transportation in the early secretory pathway [42-44]. Eimeria tenella cannot enter host cells when the secreted proteins are neutralized by monoclonal antibodies [45], and/or when the cytoskeleton is blocked by drugs, such as cytochalasin D [46]. These results suggest that E. tenella motility during the invasion of host cells is an active process and depends on actin-based contractile systems regulated by different actin-binding proteins. In the present study, actin-like proteins were identified but their function in invasion needs to be further studied.

In the present study, several key enzymes that participate in energy and metabolic processes in E. maxima were found to bind to chicken jejunal epithelial cells, including lactate dehydrogenase (LDH), enolase 2 (ENO2) and aldolase (ALD). It has been reported that immunization with DNA vaccine carrying the $E$. acervulina $\mathrm{LDH}$ antigen gene could induce protective immunity against homologous infection and immunity could be enhanced by the co-expression of chicken IL-2 or IFN- $\gamma$ [47]. Recent studies demonstrated that TgALD1 was required for energy production and was essential for efficient host cell invasion, based on its ability to bridge adhesin-cytoskeleton interactions in T. gondii $[48,49]$. Another study evidenced that EtENO had a function not only in glycolysis during anaerobic intracellular stages but also in parasite invasion [50]. Plasmodium falciparum enolase was localized to the merozoite surface, indicating that PfENO might be involved in red blood cell invasion [51]. In this investigation, these proteins were found to interact with jejunal epithelial cells, but on the other hand, their exact during cell invasion by E. maxima need to be better studied.

In this study, 35 proteins binding to chicken jejunal epithelial cells contained more than two unique peptide counts, including heat-shock proteins 60,70 and 90 , elongation factor 1-alpha (EF-1 $\alpha)$ and elongation factor 2. It was reported that EF- $1 \alpha$ played an essential role in mediating host cell entry by the parasite and could be a candidate vaccine antigen [20]. Moreover, other studies indicated the heat shock protein 70 of E. tenella could stimulate the immune system and enhance the protective immunity elicited by $E$. tenella antigen microneme protein 2 (EtMIC2) against avian coccidiosis [52]. Additionally, heat-shock protein 90 was identified as crucial for E. tenella invasion and survival in host cells [53].

In the present study, Western blot analysis of proteins of co-cultured cells revealed that only four distinct protein bands were recognized using the antisera. However, in the analysis by QE shotgun LC-MS/MS, a total of 204 nonredundant proteins were identified by searching the $E$. maxima database. The difference between results obtained after using these two techniques could be explained based on their levels of sensitivity. Based on how QE shotgun LC-MS/MS works, this technique is more sensitive than conventional techniques such as Western blot.

\section{Conclusions}

In conclusion, we found that 35 proteins of E. maxima with more than two unique peptide counts identified by shotgun LC-MS/MS could bind to chicken jejunal epithelial cells. The identified proteins were annotated according to Gene Ontology for molecular function, biological process and cellular localization. Many of these proteins play important roles in parasite invasion and motility. However, investigations on the molecular mechanisms of these proteins in the invasion process are warranted.

\section{Additional files}

Additional file 1: Table S1. LC-MS/MS analysis of E. maxima sporozoite proteins binding to chicken jejunal epithelial cells. This file describes the details of the 204 non-redundant proteins that were identified using shotgun LC-MS/MS. (XLS $187 \mathrm{~kb}$ )

Additional file 2: Table S2. Eimeria maxima sporozoite soluble proteins binding to chicken jejunal epithelial cells with more than two unique peptide counts. This file describes the details of the 35 proteins binding to chicken jejunal epithelial cells that were identified with more than two unique peptide counts using shotgun LC-MS/MS. (XLS 95 kb)

\section{Abbreviations}

ALD: aldolase; DAB: 3,3'-diaminobenzidine; ENO: enolase; GO: Gene Ontology; HBSS: Hanks' balanced salt solution; HRP: horseradish peroxidase; LC-MS/ MS: liquid chromatography and tandem mass spectrometry; LDH: lactate dehydrogenase; MDBK: Madin-Darby bovine kidney cells; MIC: microneme; PBS: phosphate-buffered saline; SDS-PAGE: sodium dodecyl sulfate polyacrylamide gel electrophoresis

\section{Acknowledgements}

We thank Dr Ibrahim Hassan from Shanghai Veterinary Research institute, Chinese Academy of Agricultural Sciences for kindly reading this manuscript and giving revision suggestions.

\section{Funding}

This work was funded by a grant from the National Natural Science Foundation of P.R. China (No. 31372428) and a project funded by the Priority Academic Program Development of Jiangsu Higher Education Institutions (PAPD).

Availability of data and materials

The proteomics data generated and analyzed during the current study are available in the iProX repository with ID IPX0001157001 (http://www.iprox.org// page/SDV015.html?subprojectld=IPX0001157001) and the ProteomeXchange 
Consortium repository with ID PXD009075 (http://proteomecentral. proteomexchange.org/cgi/GetDataset?ID=PXD009075).

\section{Authors' contributions}

$\mathrm{XL}$ directed the project and participated in the coordination and management of the study. JH performed the laboratory tests and the data analysis and wrote the manuscript. TL and KL helped perform the laboratory tests. RY, XS and LX provided new analytical reagents and tools. All authors read and approved the final manuscript.

\section{Ethics approval and consent to participate}

The animal handling procedures complied with the guideline of the Animal Ethics Committee of Nanjing Agriculture University, China and all animal experiments complied with the guidelines of the Animal Welfare Council of China. The experimental protocols were approved by the Science and Technology Agency of Jiangsu Province (Protocol code SYXK (SU) 2010-0005).

\section{Consent for publication}

\section{Not applicable.}

\section{Competing interests}

The authors declare that they have no competing interests.

Received: 25 October 2017 Accepted: 26 March 2018 Published online: 04 April 2018

\section{References}

1. Frolich S, Wallach M. Use of fluorescent nanoparticles to investigate nutrient acquisition by developing Eimeria maxima macrogametocytes. Sci Rep. 2016;6:29030

2. Huang J, Zhang Z, Li M, Song X, Yan R, Xu L, et al. Immune protection of microneme 7 (EmMIC7) against Eimeria maxima challenge in chickens. Avian Pathol. 2015;44:392-400.

3. Rose ME, Hesketh P. Immunity to coccidiosis: stages of the life-cycle of Eimeria maxima which induce, and are affected by, the response of the host. Parasitology. 1976:73:25-37.

4. Marquardt WC. Host and site specificity in the coccidia: a perspective. J Protozool. 1981:28:243-4

5. Morahan BJ, Wang L, Coppel RL. No TRAP, no invasion. Trends Parasitol. 2009:25:77-84.

6. Dzik JM. Molecules released by helminth parasites involved in host colonization. Acta Biochim Pol. 2006;53:33-64.

7. Lai L, Bumstead J, Liu Y, Garnett J, Campanero-Rhodes MA, Blake DP, et al. The role of sialyl glycan recognition in host tissue tropism of the avian parasite Eimeria tenella. PLoS Pathog. 2011;7:e1002296.

8. Song X, Ren Z, Yan R, Xu L, Li X. Induction of protective immunity against Eimeria tenella, Eimeria necatrix, Eimeria maxima and Eimeria acervulina infections using multivalent epitope DNA vaccines. Vaccine. 2015;33:2764-70

9. Huang J, Zhang Z, Li M, Song X, Yan R, Xu L, Li X. Eimeria maxima microneme protein 2 delivered as DNA vaccine and recombinant protein induces immunity against experimental homogenous challenge. Parasitol Int. 2015;64:408-16.

10. Hassan IA, Wang S, Xu L, Yan R, Song X, Li X. DNA vaccination with a gene encoding Toxoplasma gondii deoxyribose phosphate aldolase (TgDPA) induces partial protective immunity against lethal challenge in mice. Parasit Vectors. 2014;7:431.

11. Yuan C, He Q, Li J, Azzam MM, Lu J, Zou X. Evaluation of embryonic age and the effects of different proteases on the isolation and primary culture of chicken intestinal epithelial cells in vitro. Anim Sci J. 2015;86:588-94.

12. Zhang Z, Wang S, Huang J, Liu L, Lu M, Li M, et al. Proteomic analysis of Eimeria acervulina sporozoite proteins interaction with duodenal epithelial cells by shotgun LC-MS/MS. Mol Biochem Parasitol. 2015;202:29-33.

13. Wang $Y$, Zhou Y, Gong H, Cao J, Zhang H, Li X, et al. Functional characterization of a cystatin from the tick Rhipicephalus haemaphysaloides. Parasit Vectors. 2015:8:140.

14. Zhu L, Liu JP, Dao J, Lu K, Li H, Gu H, et al. Molecular characterization of S. japonicum exosome-like vesicles reveals their regulatory roles in parasitehost interactions. Sci Rep. 2016;6:25885.

15. Zdobnov EM, InterProScan AR. an integration platform for the signaturerecognition methods in InterPro. Bioinformatics. 2001;17:847-8.
16. Ye J, Fang L, Zheng H, Zhang Y, Chen J, Zhang Z, et al. WEGO: a web tool for plotting GO annotations. Nucleic Acids Res. 2006;34:W293-7.

17. Zhou H, Zhao Q, Das Singla L, Min J, He S, Cong H, et al. Differential proteomic profiles from distinct Toxoplasma gondii strains revealed by 2Ddifference gel electrophoresis. Exp Parasitol. 2013;133:376-82.

18. Ashburner M, Ball CA, Blake JA, Botstein D, Butler H, Cherry JM, et al. Gene ontology: tool for the unification of biology. The Gene Ontology Consortium. Nat Genet. 2000;25:25-9.

19. Jang SI, Lillehoj HS, Lee SH, Lee KW, Park MS, Cha SR, et al. Eimeria maxima recombinant Gam82 gametocyte antigen vaccine protects against coccidiosis and augments humoral and cell-mediated immunity. Vaccine. 2010;28:2980-5.

20. Matsubayashi M, Teramoto-Kimata I, Uni S, Lillehoj HS, Matsuda H, Furuya M, et al. Elongation factor-1alpha is a novel protein associated with host cell invasion and a potential protective antigen of Cryptosporidium parvum. J Biol Chem. 2013;288: 34111-20

21. Tomley FM, Bumstead JM, Billington KJ, Dunn PP. Molecular cloning and characterization of a novel acidic microneme protein (EtMIC2) from the apicomplexan protozoan parasite, Eimeria tenella. Mol Biochem Parasitol. 1996:79:195-206.

22. Carruthers VB, Giddings OK, Sibley LD. Secretion of micronemal proteins is associated with Toxoplasma invasion of host cells. Cell Microbiol. 1999:1:225-35.

23. Bansal A, Singh S, More KR, Hans D, Nangalia K, Yogavel M, et al. Characterization of Plasmodium falciparum calcium-dependent protein kinase 1 (PfCDPK1) and its role in microneme secretion during erythrocyte invasion. J Biol Chem. 2013;288:1590-602.

24. Tomley FM, Soldati DS. Mix and match modules: structure and function of microneme proteins in apicomplexan parasites. Trends Parasitol. 2001;17:81-8.

25. Carruthers VB, Sibley LD. Sequential protein secretion from three distinct organelles of Toxoplasma gondii accompanies invasion of human fibroblasts. Eur J Cell Biol. 1997:73:114-23.

26. Singh AP, Puri SK, Chitnis CE. Antibodies raised against receptor-binding domain of Plasmodium knowlesi Duffy binding protein inhibit erythrocyte invasion. Mol Biochem Parasitol. 2002;121:21-31.

27. Carruthers VB, Tomley FM. Microneme proteins in apicomplexans. Subcell Biochem. 2008;47:33-45.

28. Friedrich N, Santos JM, Liu Y, Palma AS, Leon E, Saouros S, et al. Members of a novel protein family containing microneme adhesive repeat domains act as sialic acid-binding lectins during host cell invasion by apicomplexan parasites. J Biol Chem. 2010;285:2064-76.

29. Carruthers VB, Sibley LD. Mobilization of intracellular calcium stimulates microneme discharge in Toxoplasma gondii. Mol Microbiol. 1999;31:421-8.

30. Wallach $\mathrm{M}$. The importance of transmission-blocking immunity in the control of infections by apicomplexan parasites. Int J Parasitol. 1997;27:1159-67.

31. Belli SI, Lee M, Thebo P, Wallach MG, Schwartsburd B, Smith NC Biochemical characterisation of the 56 and $82 \mathrm{kDa}$ immunodominant gametocyte antigens from Eimeria maxima. Int J Parasitol. 2002;32:805-16.

32. Wallach M. Eimeria maxima gametocyte antigens: potential use in a subunit maternal vaccine against coccidiosis in chickens. Vaccine. 1995;13:347-54.

33. Fu H, Subramanian RR, Masters SC. 14-3-3 proteins: structure, function, and regulation. Annu Rev Pharmacol Toxicol. 2000;40:617-47.

34. Aitken A. 14-3-3 proteins: a historic overview. Semin Cancer Biol. 2006:16:162-72.

35. Meng M, He S, Zhao G, Bai Y, Zhou H, Cong H, et al. Evaluation of protective immune responses induced by DNA vaccines encoding Toxoplasma gondii surface antigen 1 (SAG1) and 14-3-3 protein in BALB/C mice. Parasit Vectors. 2012;5:273.

36. Zhao N, Gong P, Cheng B, Li J, Yang Z, Li H, et al. Eimeria tenella: 14-3-3 protein interacts with telomerase. Parasitol Res. 2014;113:3885-9.

37. Sibley LD. Intracellular parasite invasion strategies. Science. 2004;304:248-53.

38. Xu J, Qin Z, Liao Y, Xie M, Li A, Cai J. Characterization and expression of an actin-depolymerizing factor from Eimeria tenella. Parasitol Res. 2008;103:263-70.

39. Chen J, Godt D, Gunsalus K, Kiss I, Goldberg M, Laski FA. Cofilin/ADF is required for cell motility during Drosophila ovary development and oogenesis. Nat Cell Biol. 2001;3:204-9.

40. Bamburg JR. Proteins of the ADF/cofilin family: essential regulators of actin dynamics. Annu Rev Cell Dev Biol. 1999;15:185-230.

41. Nakano K, Mabuchi I. Actin-depolymerizing protein Adf1 is required for formation and maintenance of the contractile ring during cytokinesis in fission yeast. Mol Biol Cell. 2006:17:1933-45.

42. Fucini RV, Chen JL, Sharma C, Kessels MM, Stamnes M. Golgi vesicle proteins are linked to the assembly of an actin complex defined by mAbp1. Mol Biol Cell. 2002;13:621-31. 
43. Durán JM, Valderrama F, Castel S, Magdalena J, Tomás M, Hosoya H, et al. Myosin motors and not actin comets are mediators of the actin-based Golgito-endoplasmic reticulum protein transport. Mol Biol Cell. 2003;14:445-59.

44. Chen JL, Lacomis L, Erdjument-Bromage H, Tempst P, Stamnes M. Cytosolderived proteins are sufficient for Arp2/3 recruitment and ARF/coatomerdependent actin polymerization on Golgi membranes. FEBS Lett. 2004;566:281-6.

45. Uchida T, Kikuchi K, Takano H, Ogimoto K, Nakai Y. Monoclonal antibodies inhibiting invasion of cultured cells by Eimeria tenella sporozoites. J Vet Med Sci. 1997:59:721-3.

46. Bumstead J, Tomley F. Induction of secretion and surface capping of microneme proteins in Eimeria tenella. Mol Biochem Parasitol. 2000;110:311-21.

47. Song $H$, Yan $R$, Xu L, Song X, Shah MA, Zhu H, et al. Efficacy of DNA vaccines carrying Eimeria acervulina lactate dehydrogenase antigen gene against coccidiosis. Exp Parasitol. 2010;126:224-31.

48. Starnes GL, Coincon M, Sygusch J, Sibley LD. Aldolase is essential for energy production and bridging adhesin-actin cytoskeletal interactions during parasite invasion of host cells. Cell Host Microbe. 2009:5:353-64.

49. Bosch J, Buscaglia CA, Krumm B, Ingason BP, Lucas R, Roach C, et al. Aldolase provides an unusual binding site for thrombospondin-related anonymous protein in the invasion machinery of the malaria parasite. Proc Natl Acad Sci USA. 2007;104:7015-20.

50. Labbe M, Peroval M, Bourdieu C, Girard-Misguich F, Pery P. Eimeria tenella enolase and pyruvate kinase: a likely role in glycolysis and in others functions. Int J Parasitol. 2006;36:1443-52.

51. Bhowmick IP, Kumar N, Sharma S, Coppens I, Jarori GK. Plasmodium falciparum enolase: stage-specific expression and sub-cellular localization. Malar J. 2009;8:179

52. Zhang L, Ma L, Liu R, Zhang Y, Zhang S, Hu C, et al. Eimeria tenella heat shock protein 70 enhances protection of recombinant microneme protein MIC2 subunit antigen vaccination against $E$. tenella challenge. Vet Parasitol. 2012;188:239-46.

53. Shen X, Wang C, Zhu Q, Li T, Yu L, Zheng W, et al. Effect of the diclazuril on Hsp90 in the second-generation merozoites of Eimeria tenella. Vet Parasitol. 2012;185:290-5.

\section{Submit your next manuscript to BioMed Central and we will help you at every step:}

- We accept pre-submission inquiries

- Our selector tool helps you to find the most relevant journal

- We provide round the clock customer support

- Convenient online submission

- Thorough peer review

- Inclusion in PubMed and all major indexing services

- Maximum visibility for your research

Submit your manuscript at www.biomedcentral.com/submit

) Biomed Central 